\title{
Virtuelle Lernplattform fördert Lernprozesse: Ein Blick darauf, was guten Unterricht ausmacht
}

\author{
Luisa Wottrich, Christian Fischer
}

University of Tübingen

Abstract: Die Khan Academy ist eine der weltweit am meisten genutzten virtuellen Lehr-Lernplattformen. Die Lernumgebung geht auf den individuellen Wissensstand der Kinder und Jugendlichen ein und kann so die Tiefenstrukturen des Lernens fördern. Das sind Lehr-Lernprozesse, die die Lern- und Entwicklungsprozesse der Schülerinnen und Schüler am stärksten fördern. Dass die Kurse den Schülerinnen und Schülern beim Lernen von Mathematik und Naturwissenschaften helfen können, wurde bereits in einigen Studien nachgewiesen.

Angefangen hat alles mit gewöhnlichem Nachhilfeunterricht für eine 12-Jährige: Weil seine Cousine Nadia Schwierigkeiten in Mathematik hatte, begann Salman Khan ihr im Jahr 2004 Nachhilfe zu geben. Nachdem sich weitere Verwandte seinem Unterricht begeistert angeschlossen hatten, stellte Khan zwei Jahre später sein erstes Mathe-Tutorial auf YouTube ein. Was klein anfing, wurde immer größer: Schon im Jahr 2008 erreichte Khan mit seinen Lehrfilmen monatlich mehrere zehntausend Kinder und Jugendliche weltweit und gründete daraufhin die Lehr-Lernplattform Khan Academy (Infobox 1), mit dem Ziel, ein kostenloses Lernangebote für möglichst viele Lernende zur Verfügung zu stellen.

Mittlerweile werden die Kurse der Khan Academy in 40+ Sprachen in unterschiedlichen Fachrichtungen wie Mathematik, Biologie, Informatik oder Wirtschaft angeboten und von Lernenden auf der ganzen Welt genutzt (Khan Academy, 2021). Während im traditionellen Schulsystem oftmals alle Kinder im gleichen Tempo lernen, können sich die Schülerinnen und Schüler die Inhalte in den Fächern der Khan Academy selbst erarbeiten und dort anknüpfen, wo sie ihre Lücken haben. Denn: Nicht jedes Kind lernt gleich schnell. 
Manche Schülerinnen und Schüler sind gelangweilt, wenn es ihnen nicht schnell genug geht, andere wiederum gehen verloren, weil sie trotz Wissenslücken den nächsten Lernschritt mitgehen müssen. Dass die Khan Academy genau an diesem Punkt ansetzt, macht die OnlinePlattform zu einer vielversprechenden Ergänzung, sowohl im Homeschooling als auch im traditionellen Unterricht (Engler et al., 2020; Ferdinand et al., 2020).

Infobox 1. So funktioniert die Khan Academy.

Die Inhalte der Khan Academy sind in sogenannten Kursen aufgebaut und beinhalten Videos, Texte, sowie Übungen und mitunter auch Abschlusstests. Die Plattform zeichnet sich durch ein individuelles Lernangebot aus, bei dem Lernende adaptiv in ihren Lernprozessen unterstützt werden, zum Beispiel durch ein sofortiges Feedback beim Bearbeiten von Aufgaben sowie individuell angepasste Hinweise und Übungsvorschläge. Für Lehrkräfte bietet die Khan Academy die Möglichkeit, mit dem Lernfortschritt der Schülerinnen und Schüler zu interagieren. Sie können ihnen Inhalte zuteilen und diese je nach Wissenstand individuell und zeitlich anpassen. Auch die Ergebnisse und Notizen der Schülerinnen und Schüler können eingesehen werden. Dadurch erhalten Lehrkräfte ein direktes Feedback, welche Fragen den Schülerinnen und Schülern Schwierigkeiten bereiten. Auf diese Weise unterstützt die Khan Academy die instruktiven Prozesse des Lernens.

\section{Effektiver Unterricht: Der Einfluss von Tiefenstrukturen}

Was guten Unterricht ausmacht, lässt sich nicht in wenigen Sätzen beantworten. Deshalb hat es sich bewährt, zunächst zwischen Sicht- und Tiefenstrukturen des Unterrichts zu unterscheiden. Sichtstrukturen beschreiben direkt beobachtbare Merkmale des Unterrichts, zum Beispiel ob eine Aufgabe in Gruppenarbeit oder Einzelarbeit gelöst wird. Tiefenstrukturen dagegen stehen für die Lehr-Lernprozessen, die im Unterricht stattfinden. Zu den Basisdimensionen der Tiefenstrukturen gehören das Potenzial zur kognitiven Aktivierung, die konstruktive Unterstützung sowie die Klassenführung (Kunter \& Trautwein, 2013, 2013; Table 1). Studien zeigen, dass es genau diese Tiefenstrukturen sind, die die Lernund Entwicklungsprozesse der Schülerinnen und Schüler am stärksten fördern (z.B. Hattie, 
2009). Die Qualität der Interaktion zwischen einer Lehrkraft und den Schülerinnen und

Schülern oder ein direktes Feedback haben beispielsweise einen deutlich größeren Einfluss auf das Lernen als die Klassengröße oder der Einsatz bestimmter Lehrmethoden wie Frontaloder Gruppenunterricht.

Tabelle 1. Basisdimensionen effektiven Unterrichts.

\begin{tabular}{|l|l|l|}
\hline Dimension & Erklärung & Beispiele \\
\hline Klassenführung & $\begin{array}{l}\text { Die Klassenführung bezieht sich } \\
\text { vor allem auf die Störanfälligkeit } \\
\text { des Unterrichts. Es stellen sich } \\
\text { daher folgende Fragen: Wie gut } \\
\text { gelingt es einer Lehrperson, eine } \\
\text { Unterrichtseinheit so zu gestalten, } \\
\text { dass möglichst wenige Störungen } \\
\text { auftreten und alle Schülerinnen und } \\
\text { Schülern am Lernen beteiligt sind, } \\
\text { die Unterrichtszeit also möglichst } \\
\text { effektiv genutzt werden kann? }\end{array}$ & $\begin{array}{l}\text { - Regeln und Routinen werden } \\
\text { früheitig eingeführt } \\
\text { gehandhabt }\end{array}$ \\
$\begin{array}{l}\text { Unterrichtsmaterial folgt einem } \\
\text { strukturierten Plan }\end{array}$ \\
\hline $\begin{array}{l}\text { Potenzial zur } \\
\text { kognitiven } \\
\text { Aktivierung }\end{array}$ & $\begin{array}{l}\text { Inwieweit werden die Lernenden } \\
\text { angeregt, sich aktiv mit dem } \\
\text { Lernstoff auseinanderzusetzen? }\end{array}$ & $\begin{array}{l}\text { - Aufgaben, knüpfen an das } \\
\text { Vorwissen der Schülerinnen und } \\
\text { Schüler an } \\
\bullet \text { Diskurs greift Meinungen der } \\
\text { Lernenden auf } \\
\text { - Inhalte fordern heraus und lösen } \\
\text { kognitive Konflikte aus }\end{array}$ \\
\hline $\begin{array}{l}\text { Konstruktive } \\
\text { Unterstiutzung }\end{array}$ & $\begin{array}{l}\text { Die kognitive Unterstützung } \\
\text { bezieht sich auf die Art und Weise, } \\
\text { auf die Lernende Hilfe finden, } \\
\text { wenn Verständnisprobleme } \\
\text { auftreten. Dabei spielen die } \\
\text { Wertschätzung und der Respekt in } \\
\text { der Interaktion eine entscheidende } \\
\text { Rolle. }\end{array}$ & $\begin{array}{l}\bullet \text { Fehler werden konstruktiv an- und } \\
\text { aufgenommen } \\
\bullet \text { Geduld und ein angemessenes } \\
\text { Tempo }\end{array}$ \\
\hline
\end{tabular}

\section{Khan Academy fördert Tiefenstrukturen}

Zurück zur Khan Academy: Die Online-Plattform kann es Lehrkräften ermöglichen, ihre Unterrichtsangebote gemäß der Tiefenstrukturen so zu gestalten, dass die Schülerinnen 
und Schüler in ihrer kognitiven und motivational-emotionalen Entwicklung unterstützt werden.

In der Dimension der Klassenführung tragen zwei Eigenschaften der Khan Academy dazu bei, die Störanfälligkeit des Unterrichts zu reduzieren und dafür zu sorgen, dass alle Schülerinnen und Schüler am Lernen beteiligt sind. Zum einen erhalten sie ein direktes Feedback zu einer gelösten Aufgabe. Wurde eine Aufgabe falsch beantwortet, schlägt die sogenannte Just-in-Time-Hilfe entsprechende Hilfestellungen vor. Die Schülerinnen und Schüler müssen somit nicht darauf warten, dass die Lehrkraft Zeit hat, um sie in ihrem Lösungsweg zu unterstützen. Da jede Schülerin und jeder Schüler individuell an einem Computer arbeitet, kann sich zudem die „Time on Task“ erhöhen und die Schülerinnen und Schüler haben weniger Zeit, sich mit anderen Dingen zu beschäftigen oder zu stören, weil sie vielleicht mit ihren Aufgaben schon fertig sind. Für die Lehrkraft bedeutet das wiederum, dass sie weniger Zeit aufwenden muss, um Störungen zu vermeiden. Da sich die Schülerinnen und Schüler somit auch länger und intensiver mit beispielsweise mathematischen Inhalten beschäftigten, können sie auch ihre Kenntnisse in Mathematik steigern. Durch die integrierte Hilfestellung der Khan Academy gewinnen Lehrerinnen und Lehrer zudem wertvolle Zeit, individuelle Unterstützung anzubieten. Eine Studie von Daniel Light und Elizabeth Pierson zeigt, dass Lehrkräfte die Unterrichtszeit durch den Einsatz der Angebote der Khan Academy effektiver nutzen konnten (Light \& Pierson, 2014).

Die gleiche Studie weist auch auf das Potential der kognitiven Aktivierung hin. Die Basis dafür bilden Prinzipien der Gamification, nach dem die Kurse der Khan Academy aufgebaut sind und damit mehr oder weniger dem Ablauf eines Computerspiels folgen. So können durch gelöste Aufgaben und Tests Punkte gesammelt, Abzeichen gewonnen und weitere Abschnitte freigeschaltet werden. Das motiviert Schülerinnen und Schüler, sich aktiv und intensiv mit dem Lernstoff auseinanderzusetzen (Arnavut et al., 2019). Weitere Lerneinheiten werden erst dann freigeschaltet, wenn vorherige Abschnitte erfolgreich 
abgeschlossen wurden - neue Inhalte knüpfen damit immer an bereits gelerntes Vorwissen an.

Darüber hinaus haben Light und Pierson (2014) herausgefunden, dass die Schülerinnen und Schüler über mehr Vertrauen in ihre eigenen Fähigkeiten im Fach Mathematik berichteten. Das Lernen ohne Zeitdruck trägt zur konstruktiven

Unterstützung bei. Die Schülerinnen und Schüler verfolgen ihr eigenes Lerntempo und können passgenaue Unterstützung gemäß ihres individuellen Fähigkeitsniveaus erhalten. Die Visualisierung des Lernerfolgs durch die erreichten Punkte und Abzeichen verleiht den Schülerinnen und Schülern eine größere Sicherheit in Bezug auf ihr Wissen und Können und steigert damit ihr Selbstvertrauen. In weiteren Studien konnte zudem gezeigt werden, dass sich Lernende beim Lernen in der Khan Academy motivierter fühlen und ein höheres Maß an Unabhängigkeit und Selbstregulation wahrnehmen (z.B., Murphy et al., 2014; Vidergor \& Ben-Amram, 2020).

Womöglich war es also die Förderung eben jener Basisdimensionen der Tiefenstrukturen die Salman Khans Cousine Nadia trotz ihrer anfänglichen Matheschwierigkeiten, dazu befähigten, später Medizin zu studieren.

\section{References}

Arnavut, A., Bicen, H., \& Nuri, C. (2019). Students' Approaches to Massive Open Online Courses: The Case of Khan Academy. BRAIN. Broad Research in Artificial Intelligence and Neuroscience, 10, 82-90.

Engler, S., Ferdinand, J., \& Fischer, C. (2020). Khan Academy: Lernen mit einem „digitalen Nachhilfelehrer“, OSF Preprints. https://doi.org/10.31219/osf.io/kyutm

Ferdinand, J., Engler, S., \& Fischer, C. (2020). Lernen mit digitalen Lernressourcen: Beispiel Khan Academy. Schulmanagement, 5, 24-27. https://doi.org/10.31219/osf.io/stzhj 
Hattie, J. A. (2009). Visible learning: A synthesis of over 800 meta-analyses relating to achievement. New York: Routledge.

Khan Academy (2021). Millions of students lack opportunity. Together, we can give it to them. Retrieved from https://www.khanacademy.org/donors. (Accessed June 15, 2021)

Kunter, M., \& Trautwein, U. (2013). Psychologie des Unterrichts (1. Edition). Paderborn: UTB GmbH.

Light, D., \& Pierson, E. (2014). Increasing student engagement in math: The use of Khan Academy in Chilean classrooms. International Journal of Education and Development using ICT 10, 103-119.

Murphy, R., Gallagher, L., Krumm, A. E., Mislevy, J., \& Hafter, A. (2014). Research on the use of Khan Academy in schools: Research brief. Retrieved from https://www.sri. com/work/publications/research-use-khan-academy-schools-research-brief.

Vidergor, H. E., \& Ben-Amram, P. (2020). Khan academy effectiveness: The case of math secondary students' perceptions. Computers \& Education, 157, 112. https://doi.org/10.1016/j.compedu.2020.103985

Luisa Wottrich studiert Empirische Bildungsforschung und Pädagogische Psychologie M.Sc. am Hector-Institut für Empirische Bildungsforschung der Eberhard Karls Universität Tübingen.

Christian Fischer, Ph.D. ist Tenure-Track-Professor für Educational Effectiveness am Hector-Institut für Empirische Bildungsforschung der Eberhard Karls Universität Tübingen. 